\title{
Giant Aneurysm with Stent Thrombosis: A Very Rare Complication of Stent Fracture
}

\author{
Iacopo Muraca ${ }^{1}$, Matteo Pennesi ${ }^{1}$, Fernando Scudiero ${ }^{2}$, Nazario Carrabba ${ }^{1}$, Giacomo Virgili ${ }^{3}$, \\ Filippo Bruscoli ${ }^{3}$, Angela Migliorini ${ }^{1}$, Giulia Pontecorboli ${ }^{1}$, Niccolò Marchionni ${ }^{3}$ and Renato \\ Valenti $^{1 *}$ \\ ${ }^{1}$ Interventional Cardiology Unit, Cardiovascular Department, Careggi University Hospital, Italy \\ ${ }^{2}$ Cardiology Unit, Medical Science Department, ASST Bergamo Est Bolognini Hospital Seriate, Italy \\ ${ }^{3}$ Department of Clinical and Experimental Medicine, University of Florence, Italy \\ *Corresponding author: Renato Valenti, Interventional Cardiology, Careggi University Hospital, Florence, Italy; Largo Brambilla 3, \\ 50141 Florence, Italy
}

\section{ARTICLE INFO}

Received:

Published: 幽 April 23, 2021

Citation: Iacopo M, Matteo P, Fernando S Nazario C, Renato V, et al., Giant Aneurysm with Stent Thrombosis: A Very Rare Complication of Stent Fracture. Biomed J Sci \& Tech Res 35(2)-2021. BJSTR. MS.ID.005681.

Keywords: Stent Fracture; Giant Coronary Aneurysm; Multimodality Imaging; Procedural Planning, Covered Stent; Intravascular Ultrasound

Abbreviations: RCA: Right Coronary Artery; IVUS: Intravascular Ultrasound; EES: Everolimus Eluting Stent; PTFE: Polytetrafluoroethylene; SF: Stent Fracture

\section{ABSTRACT}

Introduction: Stent fracture (SF) is an uncommon complication after coronary stent implantation. This complication can be associated with restenosis, stent thrombosis and, in rare cases, with aneurysm/pseudoaneurysm development. Considering the low incidence of coronary aneurysms associated with SF, their treatment may prove challenging and needs careful planning, either we choose percutaneous or surgical approach.

Case Presentation: We present the case of a 77-year-old male with inferior STsegment elevation myocardial infarction. At coronary angiography proximal stent thrombosis of right coronary artery was found and SF with very long displacement was identified. After vessel recanalization with rheolytic thrombectomy, using a multimodality imaging, a giant coronary aneurysm was diagnosed. We proceeded to percutaneous correction and, with intravascular ultrasound guidance, a long everolimus eluting stent was implanted bridging proximal and distal necks of the aneurysm. This created a supporting structure and a safe landing zone for two overlapping covered stents, which effectively sealed the aneurysm. The CT-scan showed total exclusion of the aneurysm and good patency of the stents.

Conclusion: A complete SF (type III or IV) can be associated to coronary aneurysm. An optimal procedural management of this complication allowed a successful technical result and good clinical outcome.

\section{Introduction}

Stent fracture (SF) represents a rare complication after coronary stent implantation. This complication is commonly associated with restenosis/reocclusion or late/very late stent thrombosis; conversely cases of stent fracture associated with aneurysms or pseudoaneurysm are rarely reported. Low incidence of this complication can lead to a very challenging diagnosis. Intravascular imaging in these situations can be useful to promptly achieve the diagnosis and even more in the planning of an appropriate interventional strategy with the aim to obtain an optimal result and to improve patient's outcome.

\section{History of Presentation}

A 77-year-old patient, previously treated with multivessel percutaneous revascularization, presented with inferior STsegment elevation myocardial infarction. In his medical history there was multiple coronary risk factors, including hypertension, hyperlipidemia, type- 2 diabetes mellitus, smoking history, chronic kidney disease, peripheral artery disease; furthermore, the patient was affected by paroxysmal atrial fibrillation on oral anticoagulant therapy. In the CathLab, proximal stent thrombosis of right coronary artery (RCA) (Figure 1A) and good patency of previously implanted 
stents on the left coronary artery were detected. After comparison with previous procedure, it was possible to identify the presence of a stent fracture with very long displacement (Figure 1B).

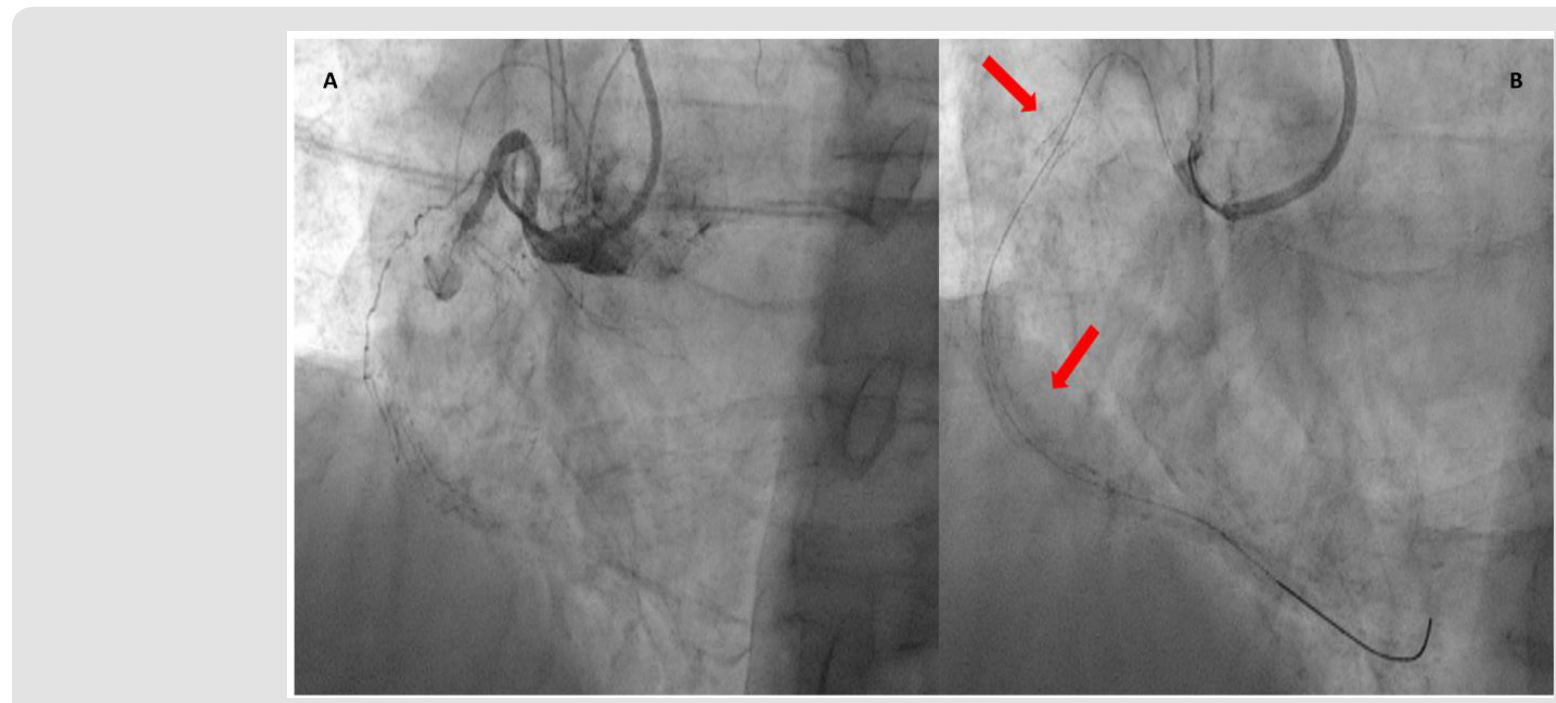

Figure 1: Proximal stent thrombosis of right coronary artery (A); evidence of stent fracture (red arrows) with very long displacement (B).

\section{Management}

Using "mother-and-child" extension catheter technique to maximize backup due to shepherd crook origin of RCA, we performed rheolytic thrombectomy and, after vessel recanalization, the presence of a massive thrombotic occlusion with wide spread of contrast dye was identified (Figure 2). Our diagnostic hypotheses were:

a) The possible presence of a giant coronary aneurysm/ pseudoaneurysm or

b) The possible rupture of the vessel wall.

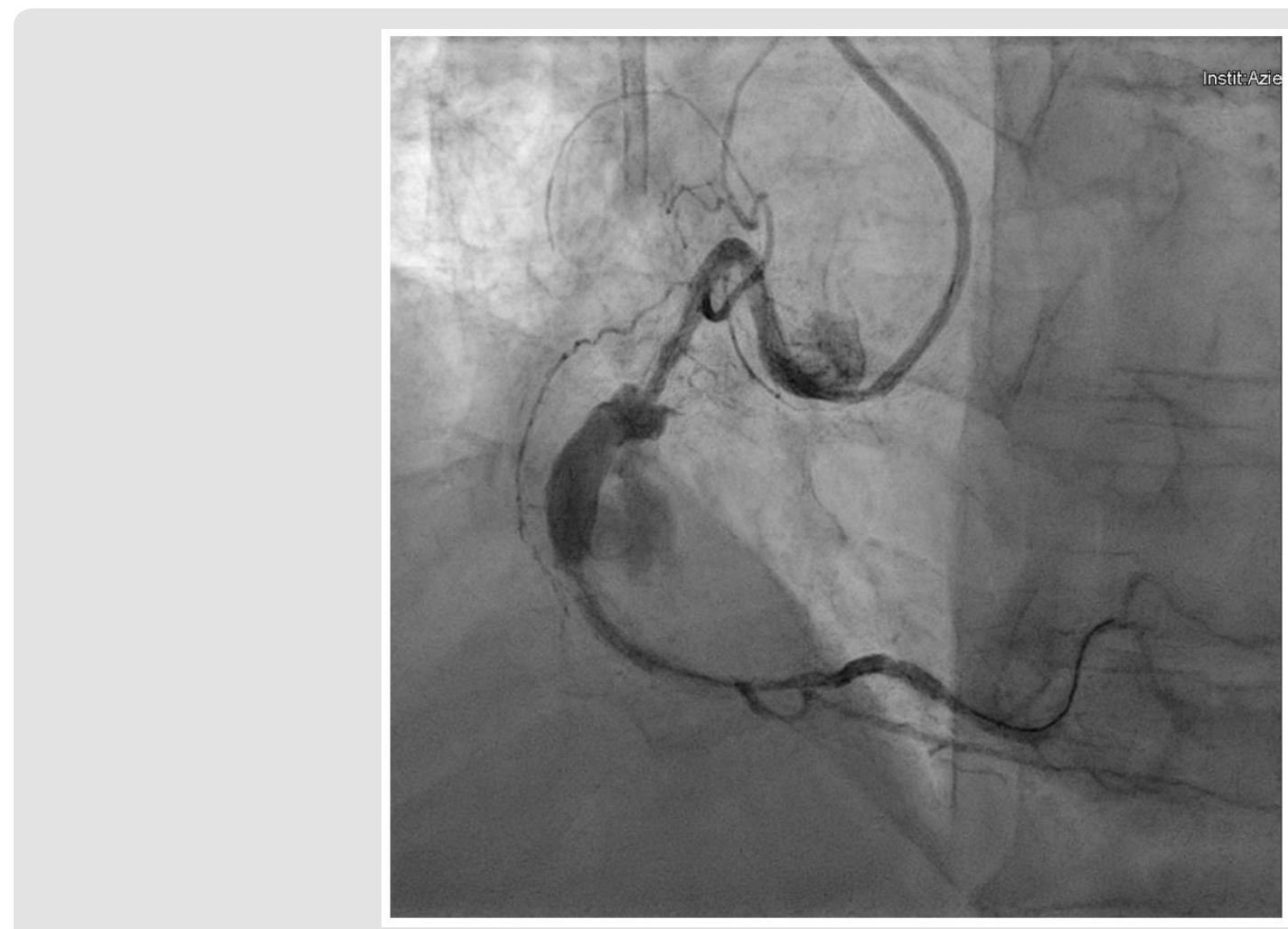

Figure 2: Wide spread of contrast dye in mid-RCA, outlining a giant aneurysm. 
Indeed, both complications can occur in case of stent fracture. Absence of pericardial effusion by echography and use of intravascular ultrasound (IVUS) imaging suggested the presence of a giant coronary aneurysm and subsequently the percutaneous repair was planned.

We performed IVUS guided implantation of a $48 \mathrm{~mm}$ long everolimus eluting stent (EES), bridging proximal and distal aneurysm necks, where previous fractured stent segments were visible, to create a supporting scaffold in order to provide a safe landing zone for deploying multiple covered stents (Figure 3). We implanted two overlapping $3.5 \times 24 \mathrm{~mm}$ single-layer polytetrafluoroethylene covered stents (BeGraft, Bentley InnoMed GmbH, Lotzenäcker 3, 72379 Hechingen, Germany), being 24 mm the maximum available length. We implanted firstly the proximal covered stent in order to facilitate tracking of the second one and to avoid the dislodgement of stiff covered stents. After the deployment of covered stents, the aneurysm was successfully excluded (Figure 4). Intra-hospital course was unremarkable. CT-scan showed a 3.5 $\mathrm{cm}$ width, $4.4 \mathrm{~cm}$ long aneurysm involving the middle segment of RCA. Contrast injection showed total exclusion of the aneurysm and good patency of implanted stents (Figure 5). One-year follow-up was uneventful.

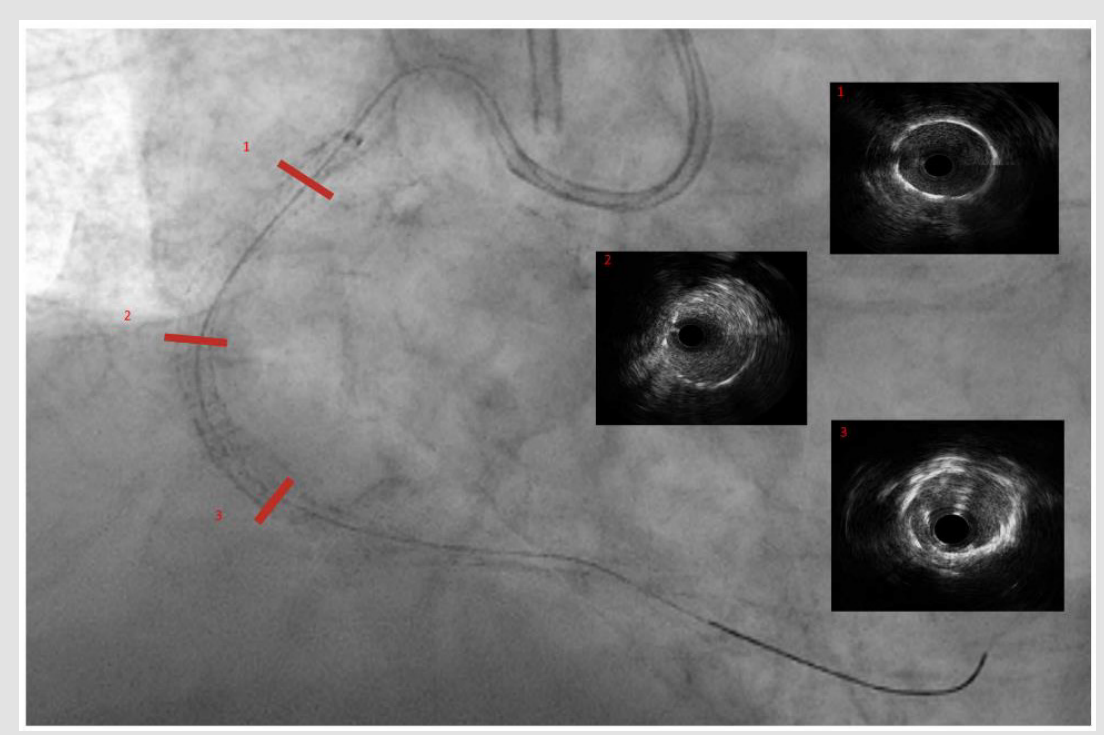

Figure 3: IVUS guided implantation of a long drug eluting stent covering aneurysm edges. Evidence of stent's malapposition in the middle part of the aneurysm (Box 2).

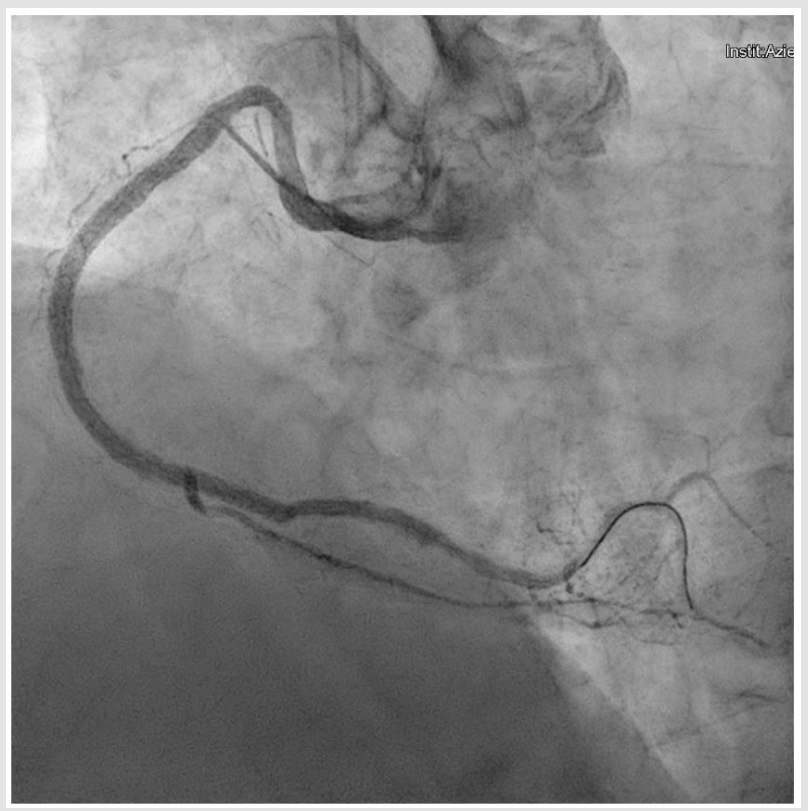

Figure 4: Final result. 


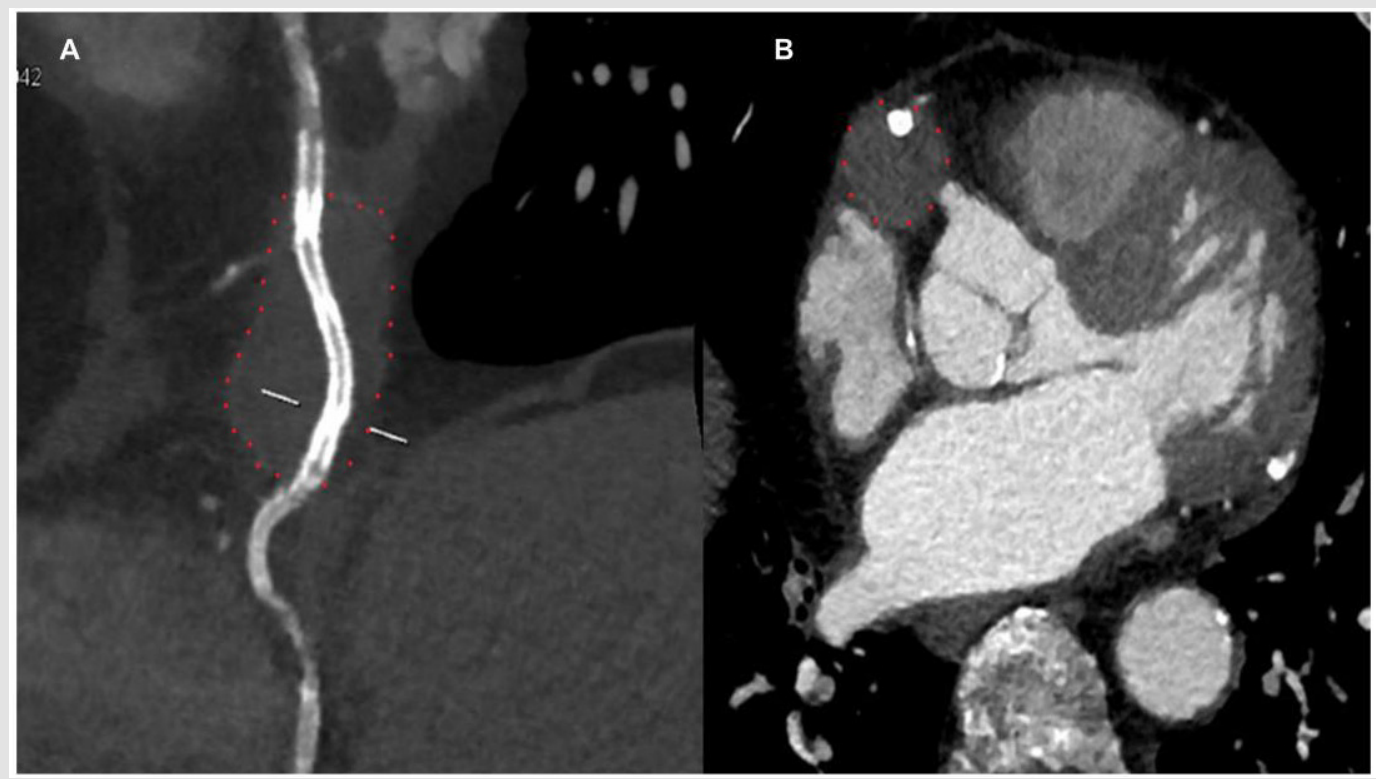

Figure 5: CT-scan showed good patency of covered stents (A); Contrast dye injection allowed to confirm complete sealing of the $3.5 \mathrm{~cm}$ aneurysm (B).

\section{Discussion}

SF is an uncommon complication in new generation DES era, ranging from 1.7 to $7.7 \%$ [1]. SF is the result of the interactions between multiple factors: stent structure, stented segment length, pulsatile and non-pulsatile forces and plaque morphology. Multiple variables have been associated with an increased rate of SF: RCA lesions treated with sirolimus eluting stents (SES), stent length, stents in angulated/tortuous vessels (hinge points), calcific lesions, vein grafts, and overlapping stents [1]. The clinical presentation of a $\mathrm{SF}$ is variable, ranging from asymptomatic to sudden cardiac death. SF may result in perforation, restenosis or reocclusion, migration of the stent segments, pseudoaneurysm formation, embolization and late and very late thrombosis associated with acute coronary syndrome and poor prognosis [1]. Due to the low incidence of this complication, it becomes evident the difficulty in diagnostic and therapeutic management. In our case the SF presented in an acute setting with massive thrombosis of a giant aneurysm. The SF of the case reported, was a type IV according to Allie et al. classification [2] with a very long stent displacement. Complete SF (type III or IV) are often associated with coronary aneurysm, but a prompt identification and an "optimal" procedural management of this complication allow a successful technical result and a good clinical outcome [3]. In our patient, after the vessel recanalization, it was crucial the differential diagnosis between coronary perforation and giant coronary aneurysm. Imaging played a key role: absence of pericardial effusion by transthoracic echocardiography, and IVUS images, sustained the hypothesis of giant coronary aneurysm.

The possible therapeutic strategies included: surgical resection, balloon or stent-assisted coil embolization or covered stent exclusion [4]. In our case, in an acute setting, we opted for the covered stent exclusion, adopting a new procedural planning. The first step was the implantation of a long EES (48 $\mathrm{mm}$ ) to provide a supporting structure, to avoid covered stent dislocation inside the aneurysm/pseudoaneurysm and to prevent a new fracture due to the continuous mechanical stress. As expected, after the everolimus-eluting stent implantation there was no aneurysm sealing. The second step was the implantation of covered stents; we had to use an overlap of two stents in order to completely exclude the aneurysm, since the maximum length available of those devices was inferior to the aneurysm length. All the diagnostic and therapeutic steps were pursued with a multimodality imaging including angiography and IVUS [5].

\section{Conclusion}

$\mathrm{SF}$ is an uncommon adverse event, and its management is still not well established, given its low incidence and wide variety of presentation. In our case, the differential diagnosis between wall perforation and giant aneurysm has been crucial for the interventional procedure strategy. Multimodality imaging confirms its fundamental support for complex coronary percutaneous procedures.

\section{Disclosure}

The authors have nothing to disclose.

\section{Acknowledgment}

The authors are indebted to the Cath Lab, Operating Room and CCU staffs for their precious help. They are also very grateful to Fabio Torrini and Paola Baldini (A.R. Card Onlus Foundation; Florence; Italy) for their secretarial assistance. 


\section{References}

1. Aoki J, Nakazawa G, Tanabe K, Angela Hoye, Hirosada Yamamoto, et al. (2007) Incidence and clinical impact of coronary stent fracture after sirolimus-eluting stent implantation. Catheter Cardiovasc Interv 69(3): 380-386.

2. Adlakha S, Sheikh M, Wu J, Burket MW, Pandya U, et al. (2010) Fracture in the coronary and peripheral arteries. J Interv Cardiol 23(4): 411-419.

3. Allie D, Herbert C, Walker C (2004) Nitinol stent fractures in the SFA Endovasc Today 3: 22-34.

ISSN: 2574-1241

DOI: 10.26717/BJSTR.2021.35.005681

Renato Valenti. Biomed J Sci \& Tech Res

(C) (P) This work is licensed under Creative

Submission Link: https://biomedres.us/submit-manuscript.php
4. Kan J, Ge Z, Zhang JJ, Liu ZZ, Tian NL, et al. (2016) Incidence and Clinical Outcomes of Stent Fractures on the Basis of 6,555 Patients and 16,482 Drug-Eluting Stents From 4 Centers. JACC Cardiovasc Interv9(11): 11151123.

5. Kawsara A, Núñez Gil IJ, Alqahtani F, Moreland J, Rihal CS, et al. (2018) Management of Coronary Artery Aneurysms. JACC Cardiovasc Interv 11(13): 1211-1223.

BIOMEDICAL
RESEARCHES $\quad \begin{aligned} & \text { Assets of Publishing with us } \\ & \text { - } \text { Immediate, unrestricted online access }\end{aligned}$

